Article

\title{
Biosynthesis of Polyunsaturated Fatty Acids in the Oleaginous Marine Diatom Fistulifera sp. Strain JPCC DA0580
}

\author{
Yue Liang ${ }^{1, \dagger}$, Yoshiaki Maeda ${ }^{1, \dagger}$, Yoshihiko Sunaga ${ }^{1,2}$, Masaki Muto ${ }^{1,2}$, \\ Mitsufumi Matsumoto ${ }^{2,3}$, Tomoko Yoshino ${ }^{1}$ and Tsuyoshi Tanaka ${ }^{1,2, *}$
}

1 Division of Biotechnology and Life Science, Institute of Engineering, Tokyo University of Agriculture and Technology, 2-24-16, Naka-cho, Koganei, Tokyo 184-8588, Japan;

E-Mails: 50011702110@st.tuat.ac.jp (Y.L.); y_maeda@cc.tuat.ac.jp (Y.M.); 50010702105@st.tuat.ac.jp (Y.S.); m_muto@cc.tuat.ac.jp (M.M.); y-tomoko@cc.tuat.ac.jp (T.Y.)

2 Japan Science and Technology Agency (JST), Core Research for Evolutionary Science and Technology (CREST), 5, Sanbancho, Chiyoda-ku, Tokyo 102-0075, Japan;

E-Mail: Mitsufumi_Matsumoto@jpower.co.jp

3 Biotechnology Laboratory, Electric Power Development Co., Ltd, 1, Yanagisaki-machi, Wakamatsu-ku, Kitakyusyu 808-0111, Japan

$\dagger$ These authors contributed equally to this work.

* Author to whom correspondence should be addressed; E-Mail: tsuyo@cc.tuat.ac.jp; Tel.: +81-42-388-7401; Fax: +81-42-385-7713.

Received: 18 October 2013; in revised form: 18 November 2013 / Accepted: 20 November 2013 / Published: 11 December 2013

\begin{abstract}
Studies of polyunsaturated fatty acid (PUFA) biosynthesis in microalgae are of great importance for many reasons, including the production of biofuel and variable omega 3-long chain PUFAs. The elucidation of the PUFA biosynthesis pathway is necessary for bioengineering to increase or decrease PUFA content in certain microalgae. In this study, we identified the PUFA synthesis pathway in the oleaginous marine diatom, Fistulifera sp. strain JPCC DA0580, a promising candidate for biodiesel production. The data revealed not only the presence of the desaturases and elongases involved in eicosapentaenoic acid (EPA) synthesis, but also the unexpected localization of 133 -desaturase expression in the chloroplast. This suggests that this microalga might perform the final step of EPA synthesis in the chloroplast and not in the endoplasmic reticulum (ER) like other diatoms. The detailed fatty acid profile suggests that the EPA was synthesized only through the w6-pathway in this strain, which was also different from other diatoms. Finally, the transcriptome analysis demonstrated an overall down-regulation of desaturases and
\end{abstract}


elongases over incubation time. These genetic features might explain the decrease of PUFA percentage over incubation time in this strain. The important insights into metabolite synthesis acquired here will be useful for future metabolic engineering to control PUFA content in this diatom.

Keywords: marine oleaginous diatom; Fistulifera sp. JPCC DA0580; eicosapentaenoic acid; polyunsatulated fatty acid; desaturase

\section{Introduction}

Bioenergy production (e.g., bioethanol, biobuthanol, and biodiesel) using photosynthetic systems is widely accepted as a promising solutions to the increasing energy crisis. Among biofuels, biodiesel fuel (i.e., fatty acid methyl ester: FAME) production using microalgal biomass is an emerging technology, and has attracted increasing attention due to advantageous features for a sustainable fuel supply (e.g., capacity for $\mathrm{CO}_{2}$ fixation, independence from agriculture, and higher yield of biofuel raw materials as compared to land plants) [1].

In biodiesel production from microalgal metabolites, FAMEs are generated by methanolysis of microalgal lipids (mainly triacylglycerols and perhaps a few free fatty acids), and the FAME composition directly determines the biodiesel fuel quality. The physical and chemical properties of the FAMEs depend on the structural features of the acyl chain, e.g., carbon chain length, chain branching, and unsaturation degree. For example, the high proportion of polyunsaturated fatty acid methyl esters (PUFAMEs) will lead to less oxidative stability of the final biodiesel [2], and, thus, have been limited to less than $1 \%$ by the EN 14214 standards in Europe. Specific enzymes, i.e., desaturases and elongases are responsible for fatty acid desaturation and elongation reactions. Molecular engineering strategies have been proposed to suppress the expression of desaturase genes, to reduce the proportion of polyunsaturated fatty acids (PUFA), with some successful attempts in decreasing the unsaturation degree of lipids in terrestrial plants, such as soy beans [3-5]. However, little progress has been reported in microalgae despite tremendous efforts. This may be attributed to insufficient knowledge regarding PUFA synthesis in microalgae, except for that in some model organisms [6-8], and difficulties in genetic engineering.

Research in PUFA biosynthesis in microalgae receives much attention also because microalgae are

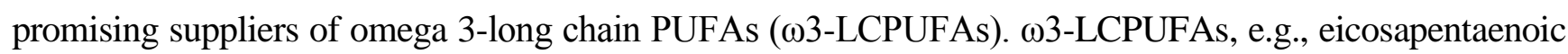
acid (EPA, C20:5n3) and docosahexaenoic acid (DHA, C22:5n3) are dietary-supplements for humans [9], with an increasing demand for production. The growing market has made it impossible to maintain a sustainable supply from the conventional source of fish oil. Some microalgal strains are well known to produce a high content of EPA and DHA [10], and thus are a potential alternative source of w3-LCPUFAs. Therefore, understanding PUFA biosynthesis in microalgae will not only benefit the regulation of PUFA content for biodiesel application but also enable a stable supply of fatty acid dietary products. 
Among eukaryotic microalgae, diatoms are well-established model organisms in terms of genomic and transgenic capabilities. A pennate diatom Phaeodactylum tricornutum has been used for basic research in both fuel and 63 -LCPUFA productions taking advantage of the available genome information [11] and easy transformation [12,13]. In P. tricornutum, thorough investigation revealed that EPA is synthesized as an end-product of PUFA synthesis via multiple metabolic networks, including the classical $\omega 6$-pathway, the classical $\omega 3$-pathway, a pathway relying on intermediates of both of these pathways and an alternative $\omega 3$-pathway involving $\Delta 9$-elongation and $\Delta 8$-desaturation [6]. However, the lipid production capacity of $P$. tricornutum is moderate, and, thus, this microalga may not be the best candidate lipid producer for practical application.

Fistulifera sp. strain JPCC DA0580 is an oleaginous diatom screened from our marine microalgal culture collection [14]. Beneficial features of this strain for biodiesel production include high neutral lipid content $(40 \%-60 \%$, w/w), high growth rate, and low unsaturation degree of the accumulated lipids [14]. Recently multi-omic analyses, including whole genome [15], transciptome, lipidome, and proteome [16] analyses, of this oleaginous strain have been launched. Based on current genomic information, a transformation technique for this strain has also been established [17]. In this study, we determined the putative pathways for PUFA biosynthesis in Fistulifera sp. strain JPCC DA0580. From the genome database, genes encoding the desaturases and elongases of this diatom were identified. Subsequently, the subcelluar localization of these enzymes was predicted with a series of bioinformatic analyses. The predicted pathway was further confirmed by the detailed fatty acid profile which was determined by direct transesterification of the dried biomass and the subsequent GC-MS analysis. Finally, the transcriptomic results well explained the variation in the fatty acid profile over incubation time. The elucidation of the PUFA synthesis pathway in Fistulifera sp. JPCC DA0580 would facilitate gene modification in this strain to regulate PUFA content for production of $\omega 3$-LCPUFAs or for biodiesel application.

\section{Materials and Methods}

\subsection{Culturing Fistulifera sp. Strain JPCC DA0580}

The marine diatom, Fistulifera sp. strain JPCC DA0580, was isolated from the junction of the Sumiyo and Yakugachi Rivers, in Kagoshima, Japan $\left(28^{\circ} 15^{\prime} \mathrm{N}, 1^{\circ} 9^{\circ} 24^{\prime}\right.$ E) [14]. Fistulifera sp. JPCC DA0580 was cultured in f/2 medium [18] (75 mg NaNO $3,6 \mathrm{mg} \mathrm{Na} \mathrm{HPO}_{4} \cdot 2 \mathrm{H}_{2} \mathrm{O}, 0.5 \mu \mathrm{g}$ vitamin B12, $0.5 \mu \mathrm{g}$ biotin, $100 \mu \mathrm{g}$ Thiamine $\mathrm{HCl}, 10 \mathrm{mg} \mathrm{Na} \mathrm{SiO}_{3} \cdot 9 \mathrm{H}_{2} \mathrm{O}, 4.4 \mathrm{mg} \mathrm{Na}$-EDTA, $3.16 \mathrm{mg} \mathrm{FeCl}{ }_{3} \cdot 6 \mathrm{H}_{2} \mathrm{O}$,

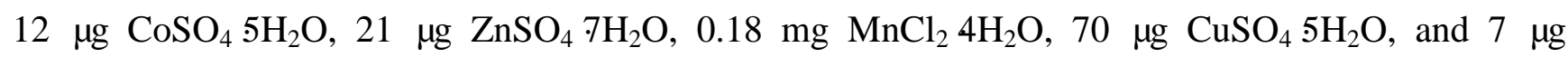
$\mathrm{Na}_{2} \mathrm{MoO}_{4} \cdot 2 \mathrm{H}_{2} \mathrm{O}$ ) dissolved per liter of artificial seawater. Cultures were aerated with sterile air at $25^{\circ} \mathrm{C}$ under $140 \mu \mathrm{mol} / \mathrm{m}^{2} / \mathrm{s}$ continuous illumination.

\subsection{Pathway Prediction}

Candidate genes involved in lipid metabolism were screened from the draft genome database of the Fistulifera sp. strain JPCC DA0580 [19], based on the Basic Local Alignment Search Tool Protein (BLASTP) search. Protein sequences with KEGG orthology (KO) number present in the Kyoto Encyclopedia of Genes and Genomes (KEGG) database or those described in published papers were 
used as query sequences. After screening, the candidate genes were submitted to InterProScan to identify protein signatures, and were subsequently annotated. In cases where gene annotations based on the protein signatures were ambiguous, phylogenetic tree analysis was also conducted to assess phylogenic relationships with diatom genes of known function. Finally, localization of proteins encoded by the annotated genes was predicted using several bioinformatics programs. TargetP [20] and HECTAR [21] were used for predicting general protein localization. Screening for endoplasmic reticulum (ER) or chloroplast-targeting peptides was performed by using SignalP [22]. When cleavage sites were predicted by SignalP, the presence of an ER retention signal $(K(/ D)-D(/ E)-E-L)$ in the protein $C$-terminus was checked manually [23]. If proteins possessed no ER retention signals and contained $\mathrm{F}, \mathrm{W}, \mathrm{Y}$, or $\mathrm{L}$ at the +1 position of cleavage sites, they were considered to be chloroplast-targeted proteins [24]. When proteins were predicted to be localized in the mitochondria by both TargetP and HECTAR, they were considered to be transported into mitochondria. In addition, Mitoprot [25] was used to detect mitochondrial-targeting peptides. If (i) the score of Mitoprot was $>0.9$ or (ii) the score of Mitoprot was $>0.8$, and mitochondrial localization was predicted by either TargetP or HECTAR, the protein was also considered to be transported into mitochondria. For proteins without signal, chloroplast-targeting, or mitochondrial-targeting peptides, peroxisome-targeting signals at the $C$-terminus (S(/A/C)-K(/R/H)-L(/M) or S-S-L) were checked manually [26]. A transmembrane prediction program (TMHMM) [27] was used to detect the presence of transmembrane regions. If transmembrane regions were predicted in proteins not containing other targeting peptides, those proteins were predicted to be localized in the ER. Proteins that conformed to none of the rules described above were predicted to be localized in the cytoplasm.

\subsection{Transcriptome Analysis}

Total RNA was extracted according to a previously published procedure [14]. In brief, cells were collected by centrifugation $\left(8500 \times \mathrm{g}, 10 \mathrm{~min}, 4^{\circ} \mathrm{C}\right)$ after 48,96 , and $144 \mathrm{~h}$ of cultivation. Cell pellets were frozen in liquid nitrogen and fractured using mortars and muddlers, and the resulting samples were treated with Plant RNA Isolation Reagent (Invitrogen Corp., Carlsbad, CA, USA) and incubated with DNase I (Takara Bio, Inc., Shiga, Japan) for $30 \mathrm{~min}$ at $37^{\circ} \mathrm{C}$. Subsequently, the total RNA was purified with the RNeasy Mini kit (Qiagen, Inc., Valencia, CA, USA). After purification, the quality of the isolated total RNA was evaluated using an Agilent 2100 Bioanalyzer (Agilent Technologies, Inc., Santa Clara, CA, USA).

For RNA sequencing, cDNA sequencing libraries were constructed using the mRNA-Seq Library Prep Kit (Illumina, Inc., San Diego, CA, USA) and the Small RNA Sample Prep Kit (Illumina, Inc., San Diego, CA, USA) according to the mRNA-Seq library preparation pre-release protocol rev.A (Illumina, Inc., San Diego, CA, USA). Briefly, poly A ${ }^{+}$RNA was isolated from $10 \mu \mathrm{g}$ of total RNA using oligo(dT) magnetic beads. The isolated poly $\mathrm{A}^{+}$RNA was fragmented into small pieces under elevated temperature. After the fragmented RNA was treated with alkaline phosphatase (Takara Bio, Inc., Shiga, Japan), the 5'-end of the fragmented RNA was phosphorylated using T4 polynucleotide kinase (Takara Bio, Inc., Shiga, Japan). Subsequently, 5'- and 3'-adapters were ligated to the phosphorylated RNA using truncated T4 RNA ligase 2 (New England Biolabs, Inc., Ipswich, MA, USA). 
The adapter-ligated RNA was reverse transcribed using primers designed for the 3 '-end adapter. The products from the reverse transcription reaction were amplified by 12 cycles of polymerase chain reaction (PCR). The resulting PCR products were size-fractionated by polyacrylamide gel electrophoresis to create a final cDNA sequencing library.

The cDNA sequencing libraries were sequenced using a Genome Analyzer IIx (Illumina, Inc., San Diego, CA, USA) according to the manufacturer's instructions. Briefly, clusters were generated from the library on the surface of a flowcell by bridge amplification using a Cluster Station (Illumina, Inc., San Diego, CA, USA) and a Single-read Cluster Generation Kit v4 (Illumina, Inc., San Diego, CA, USA). Sequencing was performed using a Genome Analyzer IIx and a TruSeq SBS kit v5 (Illumina, Inc., San Diego, CA, USA). Illumina Sequence Control Software (SCS) v2.8 with Real Time Analysis (RTA) v1.8 was used for operation and data processing, including image analysis and base calling.

Transcript abundances were expressed using the reads per kilobase of exon model per million mapped reads (RPKM) value [28]. Bowtie v0.12.7 [29] was used to align the obtained RNA-sequencing reads to the draft whole genome of Fistulifera sp. strain JPCC DA0580. ERANGE software v3.2 [28] was used to calculate RPKM values. $\log _{2}$ fold changes in RPKM values $\left(\log _{2}\left(\mathrm{RPKM}_{96 \mathrm{~h}, 144 \mathrm{~h}} / \mathrm{RPKM}_{48 \mathrm{~h}}\right)\right)$ were calculated to evaluate gene expression changes. $\mathrm{RPKM}_{48 \mathrm{~h}}$ indicates the values obtained from the mRNA sample before starting TAG accumulation, while $\mathrm{RPKM}_{96 \mathrm{~h}}$ and $\mathrm{RPKM}_{144 \mathrm{~h}}$ indicate the values obtained from mRNA samples during the TAG accumulation process. Differentially expressed genes ( $\log _{2}$ fold changes in RPKM values $\geq 1$ or $\leq-1$ ) were selected for further investigation using Gene Ontology (GO) analysis. All sequences of the differentially expressed genes in the strain were submitted to InterProScan to identify protein signatures. GO terms assigned to specific InterPro signatures were used to classify the gene products. The GO terms were classified into functional groups based on "Plant GO Slim" categories using CateGOrizer [30].

\subsection{Methyl Esterification and GC-MS Analysis}

Lyophilized microalgal cells $(25 \mathrm{mg})$ were used for methyl esterification. The dried microalgal biomass was transesterified by heating with $3 \mathrm{~mL}$ of $1.25 \mathrm{M} \mathrm{HCl}$-methanol at $90{ }^{\circ} \mathrm{C}$ for $1 \mathrm{~h}$. After methanolysis, the resulting FAMEs were extracted 3 times with $n$-hexane.

A Shimadzu GCMS QP2010 with a capillary column $(0.22 \mathrm{~mm} \times 25 \mathrm{~m})$ and a Shimadzu HiCap-CBP 5 (Shimadzu, Kyoto, Japan) were used to determine the generated FAME compositions under the following conditions: column, FAMEWAX column ( $30 \mathrm{~m}, 0.25 \mathrm{~mm} \mathrm{ID}, 0.25 \mu \mathrm{m})$; interface temperature, $240{ }^{\circ} \mathrm{C}$; injection-port temperature, $240{ }^{\circ} \mathrm{C}$; ion-source temperature, $260{ }^{\circ} \mathrm{C}$; and helium gas pressure, $60.4 \mathrm{kPa}$. Oven temperatures were programmed to $140{ }^{\circ} \mathrm{C}$ for $5 \mathrm{~min}$, then to $240{ }^{\circ} \mathrm{C}$, increasing at $4{ }^{\circ} \mathrm{C} / \mathrm{min}$, and finally to hold at $240{ }^{\circ} \mathrm{C}$ for $10 \mathrm{~min}$. FAMEs were identified by comparing the peak retention times and mass spectra of the samples with those of a standard fatty acid mixture. The GC-MS measurements were repeated three times for each sample. 


\section{Results}

\subsection{Characteristics of the Enzymes Necessary for PUFA Synthesis}

From the Fistulifera sp. strain JPCC DA0580 genome, we determined putative gene sets involved in the PUFA synthesis pathway. Fistulifera sp. possesses a complete gene set for EPA synthesis (i.e., $\Delta 9-, \Delta 12-, \Delta 6-, \Delta 5-, \omega 3$-desaturase, and $\Delta 6$-elongase). Subcellular localization of the enzymes encoded by the determined genes was also predicted by a series of bioinformatics processes. The characteristics of the putative enzymes (e.g., localization, catalytic motif, and transmembrane domains) are summarized in Table 1. The same enzymes encoded in the genomes of other diatoms, i.e., P. tricornutum [11] and Thalassiosira pseudonana [31], were also listed for comparison. Because the putative genes were determined by BLAST search based on previously identified desaturases or elongases in other organisms, it is possible that other desaturases and elongases may remain undiscovered in the Fistulifera sp. genome if they happen to have low sequence similarity to the conventional enzymes.

All desaturases determined in this study possess 3 histidine boxes, a common active site of desaturases involved in the coordination of the di-iron center [32]. Similar to the $\Delta 6-$ and $\Delta 5$-desaturases (the so-called "front-end desaturases") found in other organisms, those of Fistulifera sp. strain JPCC DA0580 also have a characteristic third histidine box, which begins with a glutamine residue instead of a histidine [33]. As we reported previously, $\Delta 9$-desaturases were divided into 2 groups: ER- and chloroplast-desaturases [34]. In this study, we determined that the $\Delta 12$-desaturases also exhibit the same characteristics. In particular, G13618 (encoded by AB858393 (g13618)) and G3281 (encoded by AB858398 ( $(3281)$ ) were predicted to localize at the ER, while G13836 (encoded by AB858392 ( $g 13836)$ ) and G14210 (encoded by AB858391 ( 14210)) were at the chloroplast. It should be noted that chloroplast $\Delta 6$-desaturases have not been predicted to be expressed by the three diatoms listed in Table 1; thus, the subcellular compartments in diatoms required for the desaturation of $\mathrm{C} 16: 2$ into $\mathrm{C} 16: 3$ are still unclear.

Furthermore, the localization prediction of all the desaturases and elongases involved in EPA synthesis in Fistulifera sp. revealed an unexpected feature that the putative $\omega 3$-desaturases localize at the chloroplast in this strain but the ER (Figure 1 and Table 1). In addition to the sequence features which were considered in our pipeline to predict protein localization (e.g., signal/transit peptides and transmembarane domains), there is another feature supporting the localization of the $\omega 3$-desaturases in the chloroplast that they have a serine/threonine-rich region after the signal peptides in the $N$-termini of these proteins [35]. In contrast to Fistulifera sp. strain JPCC DA0580, the 13 -desaturases of the other two sequenced diatoms were predicted to localize at the ER. However, other $\Delta 9-, \Delta 12-, \Delta 6-$, and $\Delta 5$-desaturases were predicted to localize at the ER in Fistulifera sp. strain JPCC DA0580. 
Table 1. Characteristics of desaturases and elongases found in the genome of Fistulifera sp. strain JPCC DA0580.

\begin{tabular}{|c|c|c|c|c|c|c|c|c|c|}
\hline \multirow{2}{*}{ Desaturase } & \multirow{2}{*}{ Organisms } & \multirow{2}{*}{ Accession No. } & \multirow{2}{*}{$\begin{array}{c}\text { Predicted } \\
\text { localization }\end{array}$} & \multirow{2}{*}{$\begin{array}{l}\text { Cytochrome } \\
\text { b5 domain }\end{array}$} & \multicolumn{3}{|c|}{ Conserved histidine boxes } & \multicolumn{2}{|c|}{ No. of predicted TMHs } \\
\hline & & & & & First & Second & Third & TMHMM & HMMTOP \\
\hline \multirow{8}{*}{$\begin{array}{l}\Delta 12(* 1) \\
\text { desaturase }\end{array}$} & \multirow[t]{4}{*}{ Fso } & AB858393 (g13618) & ER $(* 4)$ & - & HECGH & HAKHH & HVVHH & 5 & 6 \\
\hline & & AB858398 (g3281) & $\mathrm{ER}(* 4)$ & - & HECGH & НАКНН & HVVHH & 5 & 6 \\
\hline & & AB858392 (g13836) & Chloro & - & HECGH & HAVHH & HVAHH & 5 & 5 \\
\hline & & AB858391 $(g 14210)$ & Chloro & - & HECGH & HAVHH & HVAHH & 5 & 5 \\
\hline & \multirow[t]{2}{*}{ Ptr } & XM_002186103 & $\mathrm{ER}(* 4)$ & - & HECGH & HAKHН & HVVHH & 4 & 5 \\
\hline & & XM_002182796 & Chloro & - & HECGH & HAVHH & HVAHH & 5 & 5 \\
\hline & \multirow[t]{2}{*}{$T p s$} & XM_002292035 & $\mathrm{ER}(* 4)$ & - & HECGH & HAKHH & HVAHH & 4 & 2 \\
\hline & & XM_002288140 & Chloro & - & HECGH & HAVHH & HVAHH & 4 & 3 \\
\hline \multirow{4}{*}{$\omega 3$ desaturase } & \multirow[t]{2}{*}{ Fso } & AB858396 (g8143) & Chloro & - & HDAGH & HKKHH & HVIHH & 6 & 4 \\
\hline & & AB858395 ( $(g 9395)$ & Chloro & - & HDAGH & HKKHH & HVIHH & 6 & 4 \\
\hline & Ptr & XM_002185462 & $\mathrm{ER}(* 4)$ & - & HDAGH & HLKHH & HLVHH & 6 & 6 \\
\hline & $T p s$ & XM_002291021 & $\mathrm{ER}(* 4)$ & - & HDAGH & HRKHH & HVVHH & 2 & 2 \\
\hline \multirow{4}{*}{$\Delta 6$ desaturase $(* 2)$} & \multirow[t]{2}{*}{ Fso } & $\mathrm{AB} 858389(\mathrm{~g} 18158)$ & ER $(* 4)$ & + & HDFLHH & WKNKHNGHH & QVDDHHLFP & 4 & 4 \\
\hline & & AB858388 (g18269) & $\mathrm{ER}(* 4)$ & + & HDFLHH & WKNKHNGHH & QVDDHHLFP & 4 & 4 \\
\hline & Ptr & XM_002182865 & ER & + & HDFLHH & WKNKHNGHH & QVDDHHLFP & 2 & 3 \\
\hline & Tps & XM_002291493 & $\mathrm{ER}(* 4)$ & + & HDFLHH & WKNKHNGHH & QVDDHHLFP & 4 & 4 \\
\hline \multirow{6}{*}{$\Delta 5$ desaturase $(* 2)$} & \multirow[t]{2}{*}{ Fso } & AB858387 (g19653) & ER $(* 4)$ & + & HDANH & WQEQHWTHH & QVEHHLFP & 1 & 6 \\
\hline & & AB858397 ( $(g 7119)$ & $\mathrm{ER}(* 4)$ & + & HDANH & WQEQHWTHH & QVEHHLFP & 3 & 6 \\
\hline & \multirow[t]{2}{*}{ Ptr } & XM_002185696 & ER & + & HDANH & WQEQHWTHH & QVEHHLFP & 4 & 5 \\
\hline & & XM_002182822 & $\mathrm{ER}(* 4)$ & + & HDANH & WIQKHWTHH & QVEHHLFP & 4 & 6 \\
\hline & \multirow[t]{2}{*}{$T p s$} & XM_002296831 & $\mathrm{ER}(* 4)$ & + & HDANH & WLAQHWTHH & QVEHHLFP & 5 & 8 \\
\hline & & XM_002288806 & ER $(* 4)$ & + & HDANH & WMAQHWTHH & QVEHHLFP & 4 & 6 \\
\hline
\end{tabular}


Table 1. Cont.

\begin{tabular}{|c|c|c|c|c|c|c|}
\hline \multirow{2}{*}{ Elongase } & \multirow{2}{*}{ Organisms } & \multirow{2}{*}{ Accession No. } & \multirow{2}{*}{ Predicted localization } & \multirow{2}{*}{ Conserved elongase motif } & \multicolumn{2}{|c|}{ No. of predicted TMHs } \\
\hline & & & & & ТМНММ & HМMTOP \\
\hline \multirow{5}{*}{$\begin{array}{l}\Delta 6 \text { poly } \\
\text { unsaturated } \\
\text { elongase }(* 3)\end{array}$} & Fso & AB858394 (g11153) & $\mathrm{ER}(* 4)$ & QLSFLHVYHH & 5 & 7 \\
\hline & & AB858390 $(g 17615)$ & $\mathrm{ER}(* 4)$ & QLSFLHVYHH & 5 & 7 \\
\hline & Ptr & XM_002180392 & $\mathrm{ER}(* 4)$ & QLSFLHVYHH & 7 & 7 \\
\hline & & XM_002182520 & $\mathrm{ER}(* 4)$ & QLSFLHVYHH & 5 & 6 \\
\hline & $T p s$ & XM_002288445 & $\mathrm{ER}(* 4)$ & QLSFLHVYHH & 7 & 7 \\
\hline
\end{tabular}

$(* 1)$ Conserved histidine boxes are determined with the reference [35]; $(* 2)$ Conserved histidine boxes are determined with the reference [36]; $(* 3)$ Conserved elongase motifs are determined with the reference [33]; $(* 4)$ Desaturases are designated as predicted transmembrane proteins localized at the ER but not containing an ER-targeting signal peptide.

Figure 1. The fatty acid desaturation pathway in Fistulifera sp. strain JPCC DA0580, as proposed by this study. Desaturation and elongation reactions catalyzed by specific enzymes are indicated by blue arrows. Potential exchange reactions are indicated by dashed arrows. The pathways in gray letters are predicted to be inactivated in this strain due to the absence of the enzymes (interrogation marks).

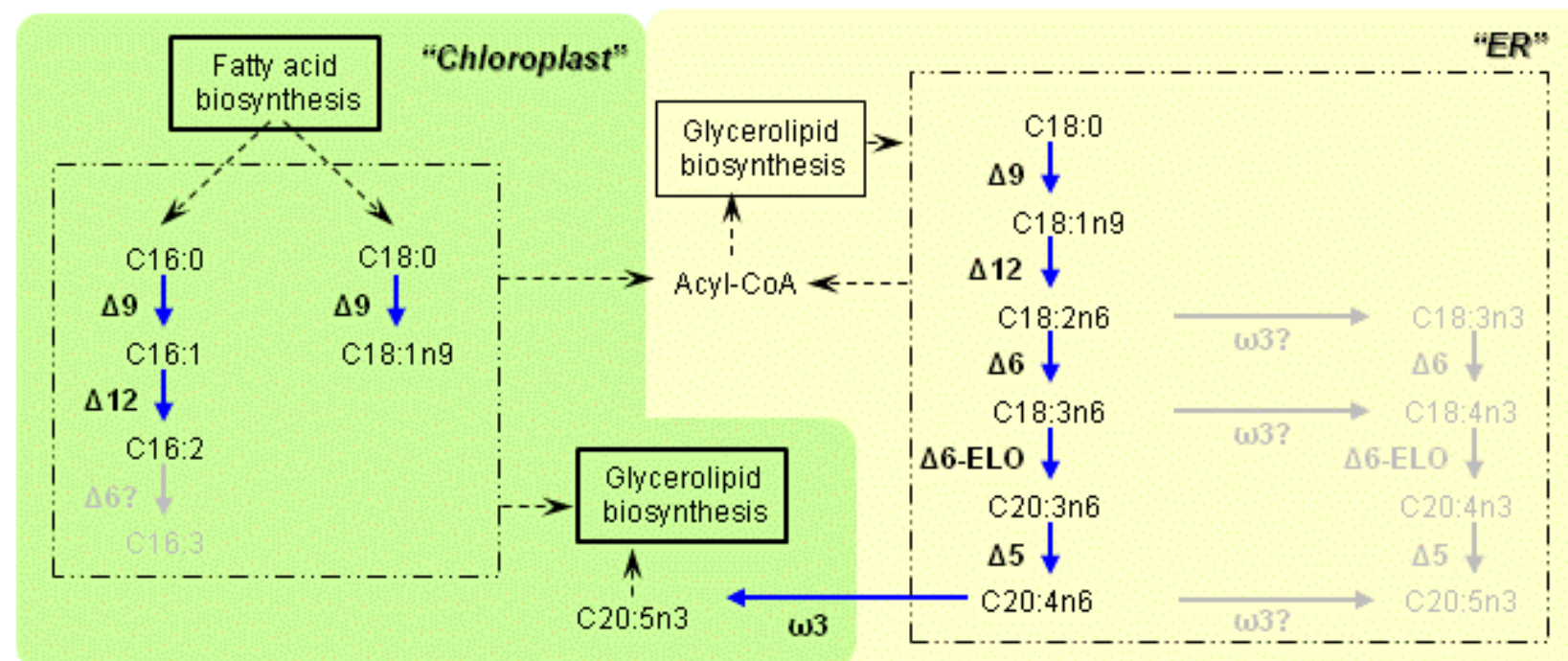




\subsection{Variation of the Fatty Acid Composition in Fistulifera sp. during Cultivation}

The composition of FAME produced from the dried cells was analyzed during cultivation with the conventional f/2 medium (Figure 2, see also Supplementary Table S1). The methyl ester forms of C16:0 and C16:1 were dominant throughout the analytical points (i.e., $48 \mathrm{~h}$, mid-log phase; $96 \mathrm{~h}$, late-log phase and $144 \mathrm{~h}$, stationary phase), and both increased during cultivation. Specifically, the proportion of $\mathrm{C} 16: 0$ was $29.8 \% \pm 0.2 \%, 37.3 \% \pm 0.2 \%$, and $36.5 \% \pm 0.0 \%$ of total FAMEs at $48 \mathrm{~h}$, $96 \mathrm{~h}$, and $144 \mathrm{~h}$, respectively, while $\mathrm{C} 16: 1$ was $36.3 \% \pm 0.3 \%, 42.4 \% \pm 0.3 \%$, and $45.9 \% \pm 0.0 \%$, at the same time-points. $\mathrm{C} 16: 2$ and $\mathrm{C} 16: 3$ were detectable in trace amounts. C20:5n3 (EPA) was the major PUFA methyl esters present, and its content decreased with incubation time $(17.0 \% \pm 0.1 \%$, $8.3 \% \pm 0.1 \%$, and $6.6 \% \pm 0.0 \%$ of total FAMEs at $48 \mathrm{~h}, 96 \mathrm{~h}$, and $144 \mathrm{~h}$, respectively). These variations over the incubation time, such as the increase of the C16:1 and C16:0 and the decrease of the C20:5, were also observed in our previous work [37], and thus considered as the representative characters of Fistulifera sp. A series of C18 and C20 fatty acid precursors for EPA synthesis (Figure 2) was confirmed, although the peaks were very small. It should be noted that all of the C18 and C20 fatty acids detected in this study were $\omega 6$-fatty acids (C18:3n6, C20:3n6 and C20:4n6), and no $\omega 3$-fatty acids (C18:3n3, C18:4n3 and C20:4n3) were detected, except for C20:5n3 (EPA).

Figure 2. Gas chromatographic analysis of total fatty acid methyl esters (FAMEs) produced from microalga biomass of Fistulifera sp. strain JPCC DA0580 at (a) $48 \mathrm{~h}$, (b) $96 \mathrm{~h}$ and (c) $144 \mathrm{~h}$ of cultivation. Fatty acids in red letters are precursors for EPA synthesis.

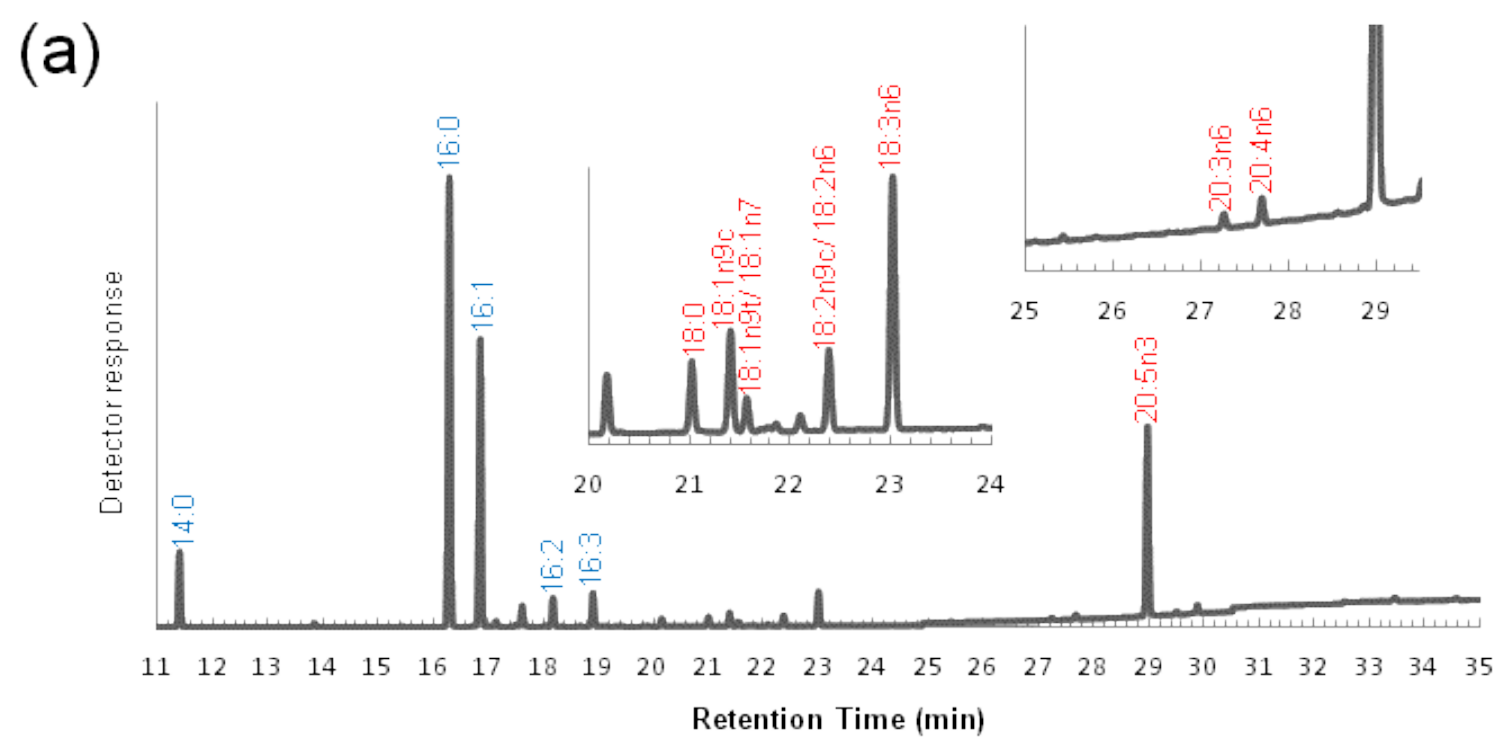


Figure 2. Cont.

(b)

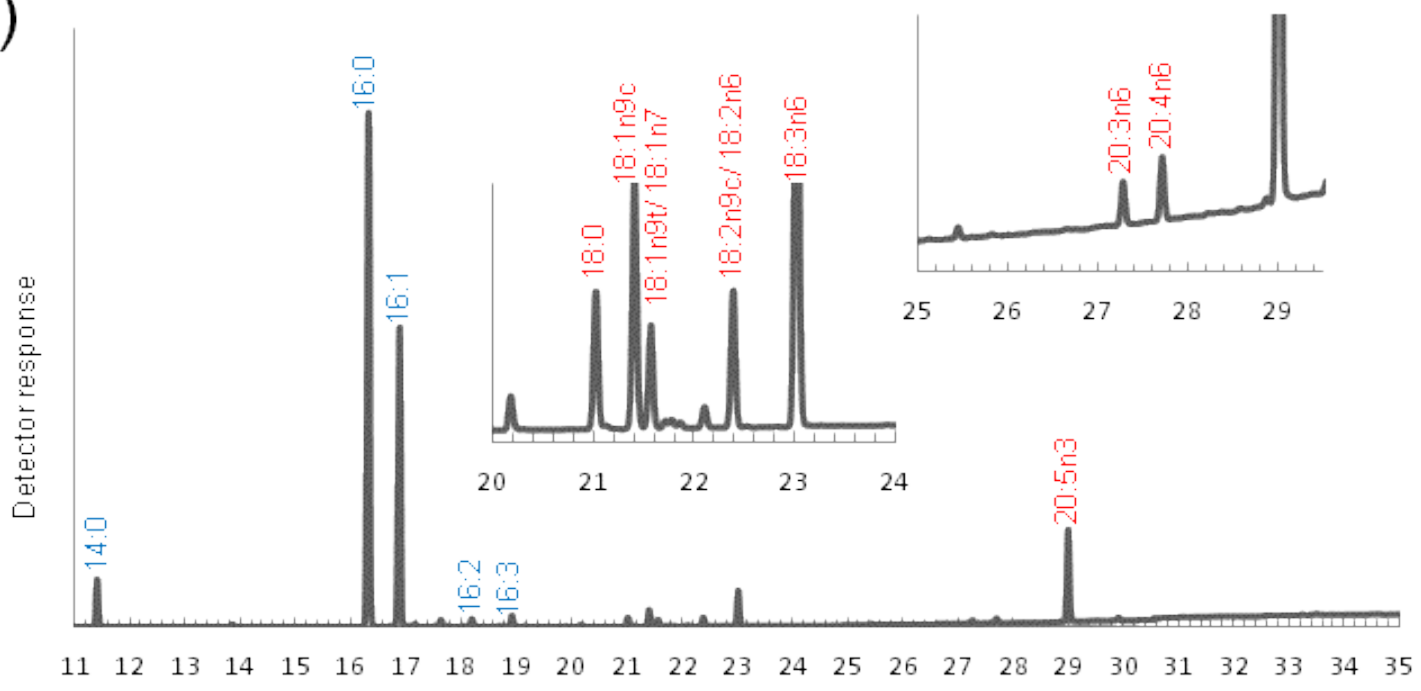

Retention Time (min)

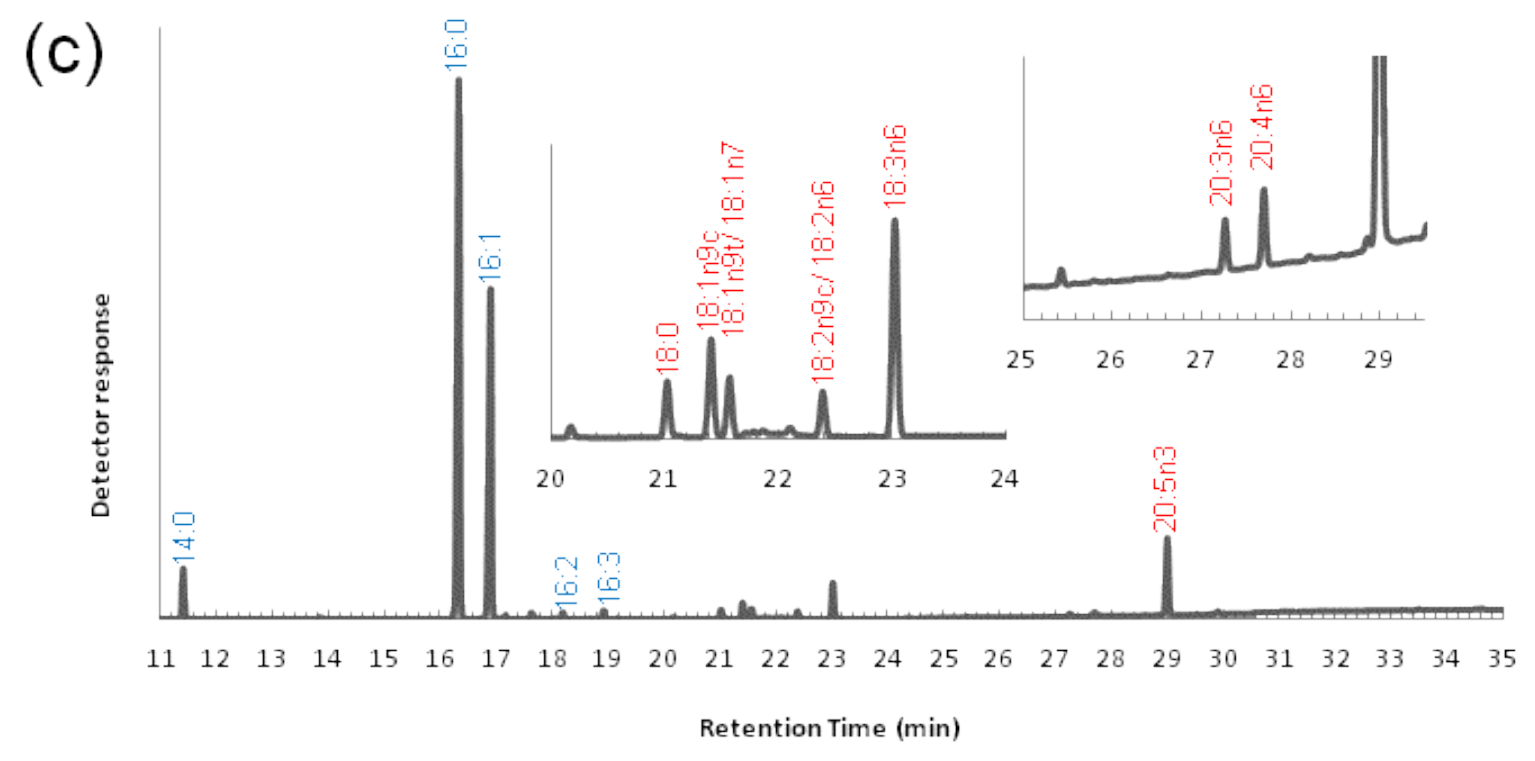

\subsection{Transcriptome Analysis of the Enzymes for Fatty Acid Desaturation}

To determine the genetic changes responsible for the FAME variation, we also analyzed the expression of the desaturases and elongases after 48 h, $96 \mathrm{~h}$ and $144 \mathrm{~h}$ of cultivation (Figure 3). During the incubation period, the $\Delta 9$-desaturases in the chloroplast, which could catalyze the conversion of C16:0 into C16:1 and C18:0 into C18:1, were the only enzymes that were up-regulated with high RPKM values. The subsequent reaction, in which C16:1 is converted into C16:2, seems to be significantly inactivated, as the $\Delta 12$-desaturases in the chloroplast had low RPKM values. Additionally, overall down-regulation of the ER-desaturases and elongases was confirmed. 
Figure 3. Genetic changes in the fatty acid desaturation pathway in Fistulifera sp. strain JPCC DA0580, as proposed by this study. Transcription activities at (a) $48 \mathrm{~h}$, (b) $96 \mathrm{~h}$ and (c) $144 \mathrm{~h}$ of cultivation are displayed as boxes. Each box represents an enzyme isoform. Sizes of the boxes and numbers in the boxes represent magnitudes of the RPKM values (3: (8) 16, 4: 32, 5: 64, 6: 128, 7: 256, 8: 512, 9: 1024, 10: 1024 ). Red boxes indicate that $\log _{2}$ fold changes in RPKM values $(96 / 48 \mathrm{~h}$ or $144 / 48 \mathrm{~h})$ were $\geq 1$. Green boxes show that $\log _{2}$ fold changes in RPKM values were $\leq-1$.

(a)

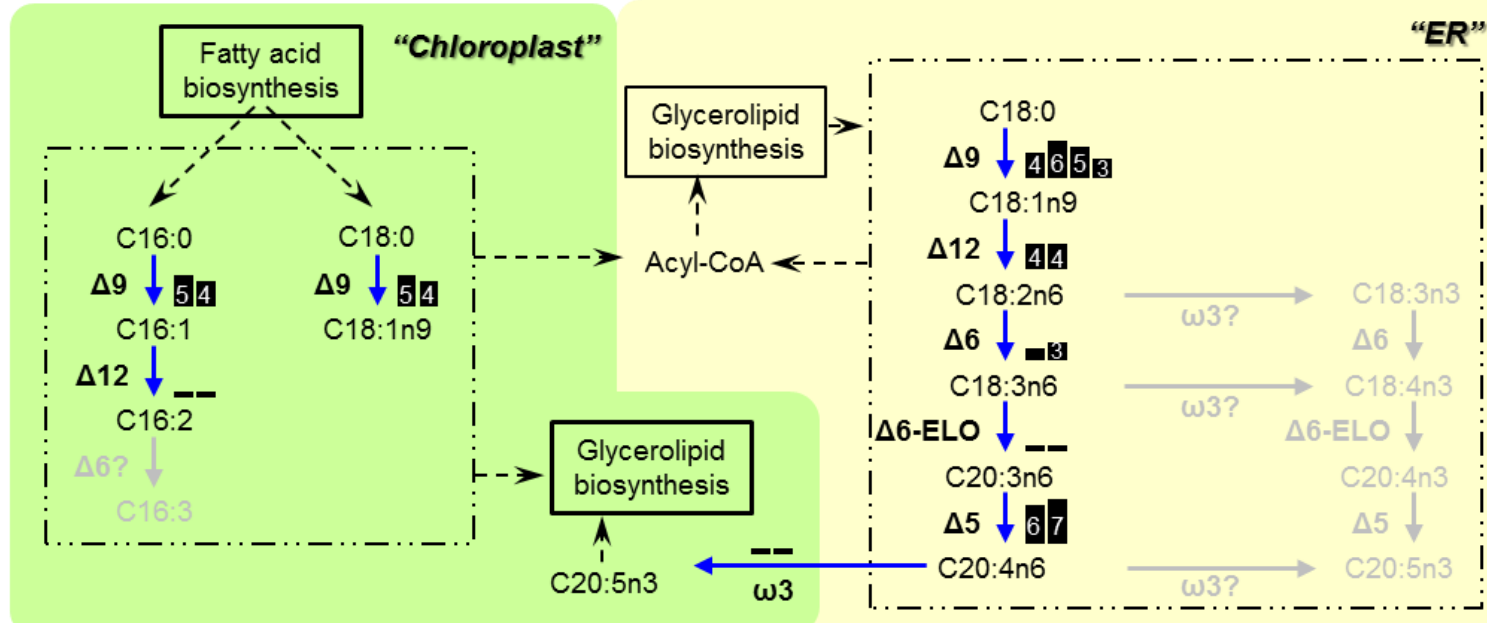

(b)

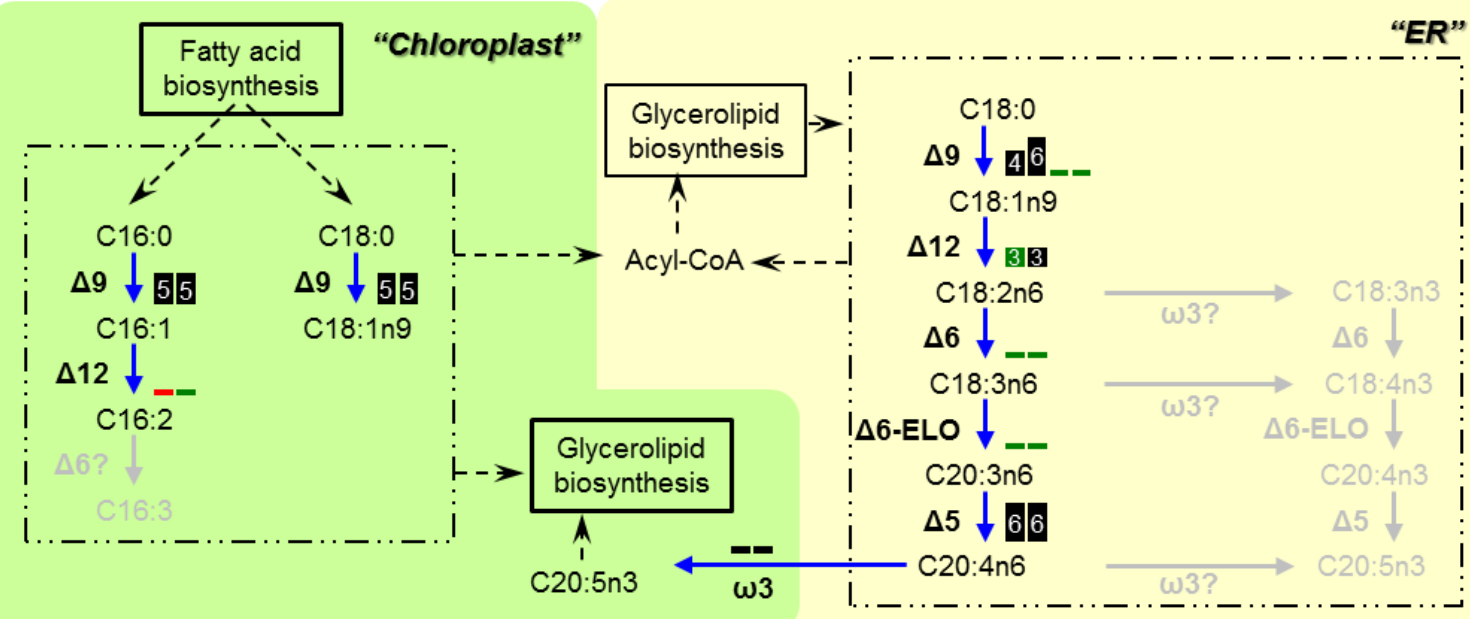

(c)

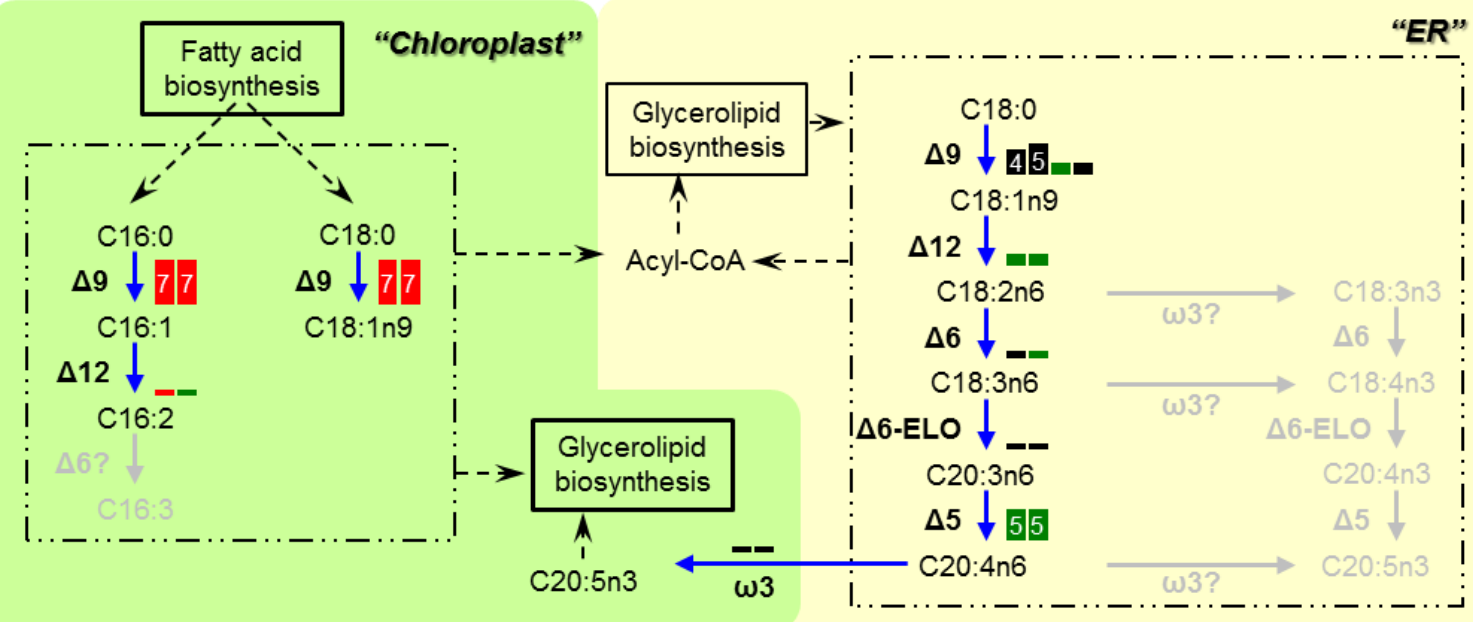




\section{Discussion}

The genomic analysis suggested that Fistulifera sp. strain JPCC DA0580 has two distinct pathways for fatty acid desaturation in the ER and chloroplast, as has been reported for other diatoms. The functions of diatom desaturases have been well studied in the diatom $P$. tricornutum, where it was demonstrated that the ER- $\Delta 12$-desaturase (encoded by XM_002186103) is highly specific to C18:1 $n 9$ while the chloroplast- $\Delta 12$-desaturases (encoded by XM_002182796) is highly specific to C16:1 [35]. The amino acid sequences of both ER- and chloroplast-desaturases of the strain JPCC DA0580 were substantially similar to their counterparts in $P$. tricornutum. The protein G13618 and G3281 ER-desaturases showed $91 \%$ similarity to the ER- $\Delta 12$-desaturase in P. tricornutum, while the G13836 and G14210 chloroplast-desaturases showed $95 \%$ and $93 \%$ similarity to the chloroplast- $\Delta 12$-desaturases in $P$. tricornutum. Therefore, it is reasonable to consider that the $\Delta 12$-desaturases in the ER and chloroplast of Fistulifera sp. would have similar substrate preferences as their counterparts in P. tricornutum. These results indicate that desaturation of $\mathrm{C} 18$ and $\mathrm{C} 16$ fatty acids mainly take place in the ER and chloroplast, respectively. Altogether, we have established a putative metabolic map for PUFA synthesis in Fistulifera sp. strain JPCC DA0580 (Figure 1).

One striking feature of the established metabolic map is the absence of $\omega 3$-desaturases in the ER, and their presence in the chloroplast. This characteristic localization has not been observed even in two other sequenced diatoms (Table 1). During EPA synthesis, the $\omega 3$-desaturase introduces a double bond at the $\omega 3$ position in the fatty acid moiety. This enzyme catalyzes the first step for EPA synthesis in the $\omega 3$ pathway, while playing a role at the final step of synthesis in the $\omega 6$ pathway (Figure 1). As no $\omega 3$-desaturase has been predicted to localize at the ER, we hypothesize that Fistulifera sp. strain JPCC DA0580 would utilize the $\omega 6$ pathway where C20:4n6 (an immediate precursor of EPA) is synthesized at the ER, with the final step in the synthesis of EPA (C20:5n3) being carried out by the $\omega 3$-desaturases in the chloroplast (Figure 1). This hypothesis is further supported by the revealed FAME profile, in which the presence $\omega 6$-fatty acids (C18:3n6, C20:3n6, and C20:4n6) was confirmed, while no $\omega 3$-fatty acids $(\mathrm{C} 18: 3 n 3, \mathrm{C} 18: 4 n 3$, and $\mathrm{C} 20: 4 n 3)$ were detected as precursors of EPA synthesis (Figure 2).

The proposed pathway depicted in Figure 1 resembles that of the red algae Porphyridium cruentum [38] rather than that of the pennate diatom $P$. tricornutum, which synthesizes EPA through both pathways in a cross-sectional manner [6]. This finding strongly suggests that the synthesis pathways for PUFAs (like EPA) are different even between the same pennate diatoms. In other words, molecular engineering efforts to increase or decrease PUFA content must target the enzymes in the appropriate pathway of each organism of interest.

The critical advantages of Fistulifera sp. strain JPCC DA0580 as a feedstock for biodiesel production include, not only the high capacity of oil accumulation [14], but also a low content of PUFA methyl esters. However, the underlying molecular mechanisms behind the beneficial low PUFA content have remained unknown. In this study, we sought clues to elucidate these underlying mechanisms via transcriptome analysis to detect key genes involved in fatty acid desaturation. As a result, the expression of the ER -enzymes in the $\omega 6$ pathway (i.e., $\Delta 9-, \Delta 12-, \Delta 6$-, and $\Delta 5$-desaturases and $\Delta 6$-elongases) was found to be globally down-regulated during incubation (Figure 3 ). This transcriptional regulation can be a reason for the decrease in EPA. Addition to EPA, the fatty acid 
analysis showed that the percentage of C18:2n6 and C20:4n6 also showed slight decrease, while other fatty acids involved in the $\omega 6$ pathway were stable over culture time (Supplementary Table S1). All those fatty acids were present in really low proportion. Low content of C16:2 and C16:3 could be another factor contributing to the low PUFA content in Fistulifera sp. strain JPCC DA0580, as other diatoms possess substantial amounts of these fatty acids [10]. These findings could also be explained by the variation in the expression of corresponding genes. As opposed to the ER- $\Delta 9$-desaturases, $\Delta 9$-desaturases of the chloroplast were up-regulated and maintained high RPKM values. However, chloroplast- $\Delta 12$-desaturases, predicted to be specific to $\mathrm{C} 16$ fatty acid based on homology analysis, were expressed at extremely low levels. These expression patterns could result in the increase of dominant C16:1 during the incubation, and also contribute to the decreased content of C16:2 and subsequent $\mathrm{C} 16: 3$. We conclude that unique transcriptional activities in the fatty acid desaturation pathways drives establishment of the characteristically low PUFA methyl ester content in Fistulifera sp. strain JPCC DA0580.

\section{Conclusions}

Based on the sequence analysis of genes which were potentially involved in the EPA synthesis, and the detailed FAME profile, we putatively determined the biosynthetic pathways for fatty acid desaturation in the oleaginous diatom, Fistulifera sp. strain JPCC DA0580. The putative pathways and their transcriptional activities were very consistent with the FAME profiles revealed by the GC-MS analysis. Interestingly, the data suggested that the metabolic pathway for EPA synthesis in Fistulifera sp. strain JPCC DA0580 was likely to be different from that in the pennate diatom $P$. tricornutum. Results from this study will be valuable to enable future metabolic engineering in order to increase EPA synthesis for commercial use, as well as to decrease poly-unsatulated FAMEs in biodiesel application.

\section{Acknowledgments}

This study was supported by Japan Science and Technology Agency (JST), Core Research for Evolutionary Science and Technology (CREST).

\section{Conflicts of Interest}

The authors declare no conflict of interest.

\section{References}

1. Hu, Q.; Sommerfeld, M.; Jarvis, E.; Ghirardi, M.; Posewitz, M.; Seibert, M.; Darzins, A. Microalgal triacylglycerols as feedstocks for biofuel production: Perspectives and advances. Plant J. 2008, 54, 621-639.

2. Chisti, Y. Biodiesel from microalgae. Biotechnol. Adv. 2007, 25, 294-306.

3. Knutzon, D.S.; Thompson, G.A.; Radke, S.E.; Johnson, W.B.; Knauf, V.C.; Kridl, J.C. Modification of Brassica seed oil by antisense expression of a stearoyl-acyl carrier protein desaturase gene. Proc. Natl. Acad. Sci. USA 1992, 89, 2624-2628. 
4. Singh, A.K.; Fu, D.Q.; El-Habbak, M.; Navarre, D.; Ghabrial, S.; Kachroo, A. Silencing genes encoding omega-3 fatty acid desaturase alters seed size and accumulation of bean pod mottle virus in soybean. Mol. Plant. Microbe Interact. 2011, 24, 506-515.

5. Liu, Q.; Singh, S.P.; Green, A.G. High-stearic and high-oleic cottonseed oils produced by hairpin RNA-mediated post-transcriptional gene silencing. Plant Physiol. 2002, 129, 1732-1743.

6. Arao, T.; Yamada, M. Biosynthesis of polyunsaturated fatty acids in the marine diatom, Phaeodactylum tricornutum. Phytochemistry 1994, 35, 1177-1181.

7. Shiran, D.; Khozin, I.; Heimer, Y.M.; Cohen, Z. Biosynthesis of eicosapentaenoic acid in the microalga Porphyridium cruentum. I. The use of externally supplied fatty acids. Lipids 1996, 31, 1277-1282.

8. Khozin, I.; Adlerstein, D.; Bigongo, C.; Heimer, Y.M.; Cohen, Z. Elucidation of the biosynthesis of eicosapentaenoic acid in the microalga Porphyridium cruentum (II. Studies with radiolabeled precursors). Plant Physiol. 1997, 114, 223-230.

9. Deckelbaum, R.J.; Torrejon, C. The omega-3 fatty acid nutritional landscape: Health benefits and sources. J. Nutr. 2012, 142, 587S-591S.

10. Arao, T.; Kawaguchi, A.; Yamada, M. Positional distribution of fatty acids in lipids of the marine diatom Phaeodactylum tricornutum. Phytochemistry 1987, 26, 2573-2576.

11. Bowler, C.; Allen, A.E.; Badger, J.H.; Grimwood, J.; Jabbari, K.; Kuo, A.; Maheswari, U.; Martens, C.; Maumus, F.; Otillar, R.P.; et al. The Phaeodactylum genome reveals the evolutionary history of diatom genomes. Nature 2008, 456, 239-244.

12. Miyahara, M.; Aoi, M.; Inoue-Kashino, N.; Kashino, Y.; Ifuku, K. Highly efficient transformation of the diatom Phaeodactylum tricornutum by multi-pulse electroporation. Biosci. Biotechnol. Biochem. 2013, 77, 874-876.

13. Apt, K.E.; Kroth-Pancic, P.G.; Grossman, A.R. Stable nuclear transformation of the diatom Phaeodactylum tricornutum. Mol. Gen. Genet. 1996, 252, 572-579.

14. Matsumoto, M.; Sugiyama, H.; Maeda, Y.; Sato, R.; Tanaka, T.; Matsunaga, T. Marine diatom, Navicula sp. strain JPCC DA0580 and marine green alga, Chlorella sp. strain NKG400014 as potential sources for biodiesel production. Appl. Biochem. Biotechnol. 2010, 161, 483-490.

15. Tanaka, T.; Fukuda, Y.; Yoshino, T.; Maeda, Y.; Muto, M.; Matsumoto, M.; Mayama, S.; Matsunaga, T. High-throughput pyrosequencing of the chloroplast genome of a highly neutral-lipid-producing marine pennate diatom, Fistulifera sp. strain JPCC DA0580. Photosynth. Res. 2011, 109, 223-229.

16. Nojima, D.; Yoshino, T.; Maeda, Y.; Tanaka, M.; Nemoto, M.; Tanaka, T. Proteomics analysis of oil body-associated proteins in the oleaginous diatom. J. Proteome Res. 2013, 12, 5293-5301.

17. Muto, M.; Fukuda, Y.; Nemoto, M.; Yoshino, T.; Matsunaga, T.; Tanaka, T. Establishment of a genetic transformation system for the marine pennate diatom Fistulifera sp. strain JPCC DA0580 - A high triglyceride producer. Mar. Biotechnol. 2013, 15, 48-55.

18. Guillard, R.R.; Ryther, J.H. Studies of marine planktonic diatoms. I. Cyclotella nana Hustedt, and Detonula confervacea (cleve) Gran. Can. J. Microbiol. 1962, 8, 229-239. 
19. Tanaka, T.; Maeda, Y.; Veluchamy, A.; Tanaka, M.; Bowler, C.; Muto, M.; Sunaga, Y.; Tanaka, M.; Yoshino, T.; Taniguchi, T.; Fukuda, Y.; Nemoto, M.; Matsumoto, M.; Wong, P.S.; Aburatani, S.; Fujibuchi, W. Whole Genome and transcriptome analyses of the oleaginous diatom Fistulifera sp. JPCC DA0580 reveal insights into simultaneous growth and oil accumulation. 2013, to be submitted for publication.

20. Emanuelsson, O.; Nielsen, H.; Brunak, S.; von Heijne, G. Predicting subcellular localization of proteins based on their $N$-terminal amino acid sequence. J. Mol. Biol. 2000, 300, 1005-1016.

21. Gschloessl, B.; Guermeur, Y.; Cock, J.M. HECTAR: A method to predict subcellular targeting in heterokonts. BMC Bioinf. 2008, 9, doi:10.1186/1471-2105-9-393.

22. Bendtsen, J.D.; Nielsen, H.; von Heijne, G.; Brunak, S. Improved prediction of signal peptides: SignalP 3.0. J. Mol. Biol. 2004, 340, 783-795.

23. Kroth, P.G.; Chiovitti, A.; Gruber, A.; Martin-Jezequel, V.; Mock, T.; Parker, M.S.; Stanley, M.S.; Kaplan, A.; Caron, L.; Weber, T.; et al. A model for carbohydrate metabolism in the diatom Phaeodactylum tricornutum deduced from comparative whole genome analysis. PLoS One 2008, 3, e1426.

24. Gruber, A.; Vugrinec, S.; Hempel, F.; Gould, S.B.; Maier, U.-G.; Kroth, P.G. Protein targeting into complex diatom plastids: Functional characterisation of a specific targeting motif. Plant Mol. Biol. 2007, 64, 519-530.

25. Claros, M.G.; Vincens, P. Computational method to predict mitochondrially imported proteins and their targeting sequences. Eur. J. Biochem. 1996, 241, 779-786.

26. Gonzalez, N.H.; Felsner, G.; Schramm, F.D.; Klingl, A.; Maier, U.G.; Bolte, K. A single peroxisomal targeting signal mediates matrix protein import in diatoms. PLoS One 2011, 6, e25316.

27. Krogh, A.; Larsson, B.; von Heijne, G.; Sonnhammer, E.L. Predicting transmembrane protein topology with a hidden Markov model: Application to complete genomes. J. Mol. Biol. 2001, 305, 567-580.

28. Mortazavi, A.; Williams, B.A.; McCue, K.; Schaeffer, L.; Wold, B. Mapping and quantifying mammalian transcriptomes by RNA-Seq. Nat. Methods 2008, 5, 621-628.

29. Langmead, B.; Trapnell, C.; Pop, M.; Salzberg, S.L. Ultrafast and memory-efficient alignment of short DNA sequences to the human genome. Genome Biol. 2009, 10, R25.

30. Zhi-Liang, H.; Bao, J.; Reecy, J. CateGOrizer: A web-based program to batch analyze gene ontology classification categories. Online J. Bioinf. 2008, 9, 108-112.

31. Armbrust, E.V.; Berges, J.A.; Bowler, C.; Green, B.R.; Martinez, D.; Putnam, N.H.; Zhou, S.; Allen, A.E.; Apt, K.E.; Bechner, M.; et al. The genome of the diatom Thalassiosira pseudonana: Ecology, evolution, and metabolism. Science 2004, 306, 79-86.

32. Los, D.A.; Murata, N. Structure and expression of fatty acid desaturases. Biochim. Biophys. Acta 1998, 1394, 3-15.

33. Hashimoto, K.; Yoshizawa, A.C.; Okuda, S.; Kuma, K.; Goto, S.; Kanehisa, M. The repertoire of desaturases and elongases reveals fatty acid variations in 56 eukaryotic genomes. J. Lipid Res. 2008, 49, 183-191. 
34. Muto, M.; Kubota, C.; Tanaka, M.; Satoh, A.; Matsumoto, M.; Yoshino, T.; Tanaka, T. Identification and functional analysis of delta-9 desaturase, a key enzyme in PUFA synthesis, isolated from the oleaginous diatom Fistulifera. PLoS One 2013, 8, e73507.

35. Domergue, F.; Spiekermann, P.; Lerchl, J.; Beckmann, C.; Kilian, O.; Kroth, P.G.; Boland, W.; Zahringer, U.; Heinz, E. New insight into Phaeodactylum tricornutum fatty acid metabolism. Cloning and functional characterization of plastidial and microsomal delta12-fatty acid desaturases. Plant Physiol. 2003, 131, 1648-1660.

36. Domergue, F.; Lerchl, J.; Zähringer, U.; Heinz, E. Cloning and functional characterization of Phaeodactylum tricornutum front-end desaturases involved in eicosapentaenoic acid biosynthesis. Eur. J. Biochem. 2002, 269, 4105-4113.

37. Satoh, A.; Ichii, K.; Matsumoto, M.; Kubota, C.; Nemoto, M.; Tanaka, M.; Yoshino, T.; Matsunaga, T.; Tanaka, T. A process design and productivity evaluation for oil production by indoor mass cultivation of a marine diatom, Fistulifera sp. JPCC DA0580. Bioresour. Technol. 2013, 137, 132-138.

38. Khozin, I.; Cohen, Z. Differential response of microalgae to the substituted pyridazinone, Sandoz 9785, reveal different pathways in the biosynthesis of eicosapentaenoic acid. Phytochemistry 1996, 42, 1025-1029.

(C) 2013 by the authors; licensee MDPI, Basel, Switzerland. This article is an open access article distributed under the terms and conditions of the Creative Commons Attribution license (http://creativecommons.org/licenses/by/3.0/). 